\title{
Single-shot measurement of a 52-fs pulse
}

\author{
F. Salin, P. Georges, G. Roger, and A. Brun
}

\begin{abstract}
We demonstrate the possibility of a 52 -fs pulse single-shot measurement by using the spatial analysis of the second harmonic beam produced in an optical autocorrelator. We show that this method gives a reliable means of optimizing low repetition rate femtosecond amplifiers.
\end{abstract}

Since the introduction of femtosecond laser systems in $1981^{1}$ much progress has been made toward reducing the duration of the produced pulses. These lasers are able to produce pulses shorter than $70 \mathrm{fs}$ and with a peak power up to $10 \mathrm{GW} .^{2,3}$ Moreover, one always uses the classical background-free autocorrelation scheme ${ }^{4}$ (modified Michelson interferometer with a scanning arm) to measure the pulses. There have not been significant advances in obtaining a real time measurement (i.e., made with a single pulse) of femtosecond pulses. In the picosecond domain, streak cameras with 0.4-ps rise time exist, but they cannot be used for 100 -fs pulses. At this time, there is much theorizing about new measurement systems for femtosecond pulses $^{5-7}$ but few experimental demonstrations of their capabilities.

The problem is to transform the temporal information (shape of the pulse) into a more convenient form (spatial or slowly varying signal). In a classical autocorrelator the delay between the two pulses is slowly swept, and the autocorrelation function is built from a large number of successive pulses. In the picosecond domain, single-shot measurements have been demonstrated using two-photon fluorescence (TPF) ${ }^{8}$ or second harmonic generation. ${ }^{9}$ In a recent paper, Sperber and Penzkofer ${ }^{8}$ show that TPF traces could be decorrelated to determine the approximate temporal shape of modulated pulses. Nevertheless, the continuous background signal produced by each individual beam gives a maximum contrast ratio of $3: 1$ between the maximum value of the autocorrelation function and the background level. In 1981, Wyatt and Marinero ${ }^{9}$

The authors are with University Paris-Sud, Institute of Theoretical and Applied Optics, UA 14 CNRS, 91406 Orsay CEDEX, France.

Received 16 November 1986.

0003-6935/87/214528-014\$02.00/0.

(C) 1987 Optical Society of America. demonstrated an original method using a diffraction grating to produce a tailored expanded beam with a differential time delay along its expanded axis. This system gives the autocorrelation function of an input pulse but suffers great losses and chromaticity. We present here the possibility of background-free singleshot measurement for pulses shorter than $100 \mathrm{fs}$. Our system is based on a method presented by Jansky et al..$^{10}$ It has already been used in the picosecond ${ }^{11}$ and subpicosecond ${ }^{12}$ domains. We show here that single 60 -fs pulses can be analyzed and that the stability of amplifiers can be studied.

The basic idea of the method is to transform the temporal shape of the pulse into a spatial shape which could be analyzed by a spatial detector. A beam splitter produces two replicas of the incident pulse which cross each other in a nonlinear crystal. The image of the crystal at the second harmonic $(\mathrm{SH})$ wavelength is recorded with a linear array of CCD. If the two incident pulses are not collinear, the $\mathrm{SH}$ signal is only produced where the pulses coincide both spatially and temporally. The exact calculation of the $\mathrm{SH}$ beam spatial shape was done by Jansky et al.,10 and we present here their results in a more intuitive way. We use Fig. 1 to obtain the intensity of the $\mathrm{SH}$ signal produced at a distance $x_{0}$ from the center of the $\mathrm{SH}$ beam. We assume that the two incident pulse intensities are spatially uniform. Let $I_{1}(t)$ and $I_{2}(t)$ be the temporal intensity shapes of the incident pulses and $S(x)$ the spatial shape of the SH signal. At a point with an abscissa $x_{0}$ (whatever its position along the $y$ direction) the instantaneous $\mathrm{SH}$ signal is proportional to $I_{1}(t-\tau) \times I_{2}(t+\tau)$ with the delay $\tau$ related to the position $x_{0}$ by

$$
\tau=\frac{n x_{0} \sin \phi / 2}{c},
$$

where $\phi$ is the angle made by the two incident beams, $n$ is the index of the nonlinear medium, and $c$ is the velocity of the light in the vacuum. 
Note that in our case $I_{1}(t)=I_{2}(t)=I(t)$. As the detector integrates the $\mathrm{SH}$ signal over a longer time than the pulse width, we finally obtain a shape $S(x)$ proportional to the second-order autocorrelation function $G_{2}(\tau)$ of the incident pulse:

$$
S(x) \propto \int_{-\infty}^{+\infty} I(t+\tau) I(t-\tau) d t=G_{2}(2 \tau) .
$$

By recording the spatial shape of the $\mathrm{SH}$ signal, one can obtain the same information as with a classical autocorrelator but in this case with a single pulse. The absolute measurement of the pulse width can be obtained by two methods.

The first is to measure the exact spatial width of the $\mathrm{SH}$ beam and to calculate the pulse width with Eqs. (1) and (2). This method implies a calibration of the detector; this is not easy for spatial width of the order of a few tens of a micrometer and needs an exact knowledge of the angle $\phi$. The second method is more attractive. It consists of the introduction of a delay $\Delta t_{0}$ into one of the two incident beams; the $\mathrm{SH}$ pattern thus shifts along the $x$ axis. This shift $\Delta x_{0}$ is related to the delay $\Delta t_{0}$ by ${ }^{10}$

$$
\Delta x_{0}=\frac{c \Delta t_{0}}{2 n \sin \phi / 2} .
$$

Having in mind that the $\mathrm{SH}$ beam shape $S(x)$ is not exactly the second-order intensity autocorrelation function $G_{2}(\tau)$ but is the related function $G_{2}(2 \tau)$, one can see that the full width at half-maximum (FWHM) $\delta_{0}$ of the spatial function $S(x)$ is connected with the temporal incident pulse FWHM $\eta_{0}$ by

$$
\eta_{0}=K \cdot \delta_{0} \frac{\Delta t_{0}}{\Delta x_{0}},
$$

where $\Delta t_{0}$ and $\Delta x_{0}$ are defined above and $K$ is a form factor depending on the incident pulse shape $(K=1.55$ for a sech ${ }^{2}$ pulse, $K=1.414$ for a Gaussian pulse, and $K$ $=1$ for a square pulse). Note that Eq. (4) shows that the calibration of this single-shot autocorrelator is obtained in exactly the same way as in a classical autocorrelator leading to a simple measurement method.

In our experiments we have used a femtosecond laser. It consists in a cw argon-pumped CPM dye laser passively mode-locked by a DODCI jet. This laser produces $50-\mathrm{fs} 0.1-\mathrm{nJ}$ pulses at $620 \mathrm{~nm} .{ }^{13}$ We use a Nd:YAG (3-ns, 10-Hz) pumped amplifier with four stages to amplify the pulses up to $500 \mu \mathrm{J}$. The pulse width at the output of the fourth stage is $\sim 400 \mathrm{fs}$ because of the amplifier group velocity dispersion. This dispersion is compensated by four high-index prisms. ${ }^{11}$ The advantages of using these prisms are a very high transmission ratio $(>92 \%)$ and a good compactness $\left(50 \times 50 \mathrm{~cm}^{2}\right)$. With this system we have already shown that, without amplification (the YAG is stopped), a 50 -fs incident pulse has a width of $\sim 380 \mathrm{fs}$ at the output of the amplifier and can be compressed to 55 fs by the prism's compressor. ${ }^{14}$

For these measurements we used a classical autocorrelator taking advantage of the $80-\mathrm{MHz}$ recurrence frequency of our oscillator. In this paper, we deal with

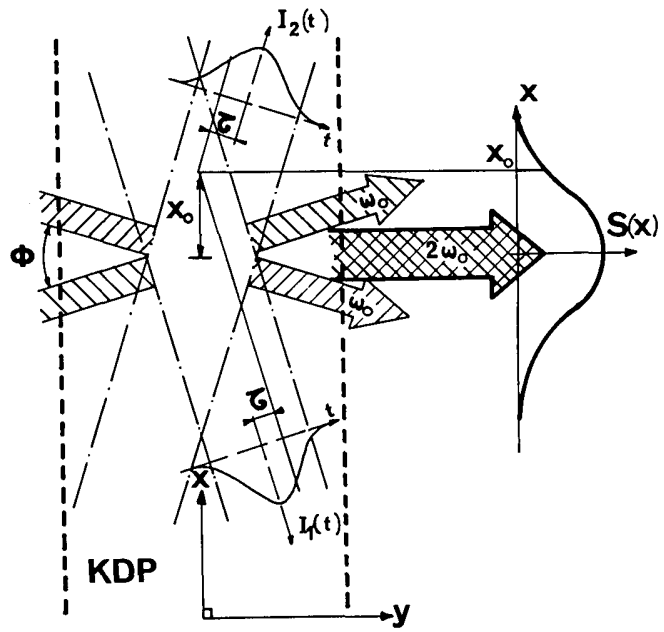

Fig. 1. Interaction of two large femtosecond beams in a nonlinear crystal.

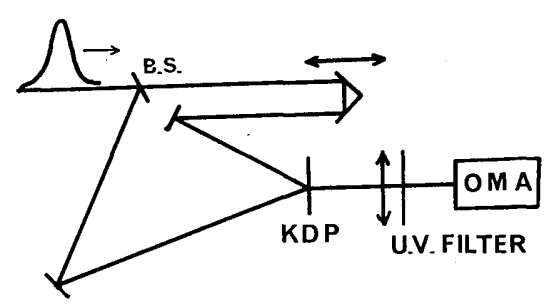

Fig. 2. Experimental setup for measurement of the $\mathrm{SH}$ beam profile.

amplified pulses. The recurrence is then $10 \mathrm{~Hz}$, and we cannot use the classical autocorrelator any more. So we built a spatial autocorrelator as sketched in Fig. 2. An incident pulse is spatially extended and split in two replicas. These two pulses overlap in a $300-\mu \mathrm{m}$ thick KDP crystal. A delay line in one arm ensures the temporal coincidence of the two pulses in the crystal and is used to calibrate the autocorrelator. We make the image of the crystal surface on an OMA I detector using a $f=50-\mathrm{mm}$ lens. A $\times 1$ magnification is used. An UV filter selects the signal at $310 \mathrm{~nm}$ and stops the incident pulses at $620 \mathrm{~nm}$. The $\mathrm{KDP}$ crystal is cut to ensure the maximum second harmonic intensity in the direction of the two incident beams' bisector.

To obtain reliable measurements uniform illumination on the crystal is essential. In our case the total power density incident on the crystal was $\sim 2 \mu \mathrm{J} / \mathrm{cm}^{2}$ with a spot size of $\sim 3 \mathrm{~cm}$. We tested the influence of the spatial beam profile on the measurement by introducing a varying delay into one arm of the autocorrelator and thus shifting the UV beam along the crystal. We found that, for our exploration range ( $\sim 10$ times the autocorrelation FWHM), the autocorrelation function keeps the same shape even with an input beam profile which is not perfectly uniform. Note that the actual width of the UV beam is $\sim 150 \mu \mathrm{m}$ for 50 -fs pulses, which limits the beam profile influence. Using this autocorrelator we measured the single-shot pulse width for different experimental conditions. First, at the output of the amplifier, we obtained 380- 


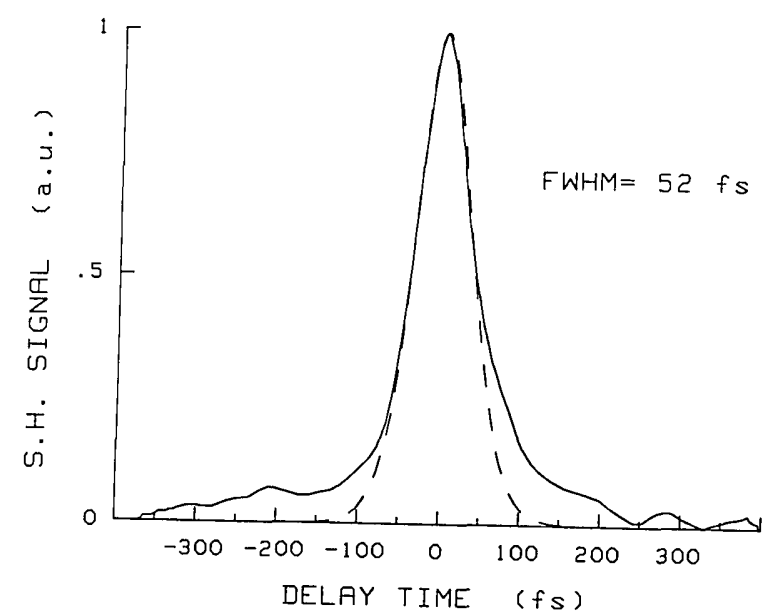

Fig. 3. Single-shot autocorrelation function (solid line) and best fit (assuming a sech ${ }^{2}$ pulse shape) giving a 52-fs FWHM (dashed line).

fs pulse widths. As the prism sequence is introduced, the pulse is compressed. Our system permits a realtime visualization of the pulse autocorrelation; therefore, the optimization of the amplifier becomes as simple as that of the CPM laser. By moving one prism, one can see the evolution of the pulse temporal shape and can easily find the best position. This allows us to obtain reliable pulses from one day to the next. During these optimizations we have found that the pulse width depends critically on the rotation of one of the prisms but that a 1-mm translation of this prism perpendicular to its base does not affect its duration dramatically. In the same way we have observed that the pulse width at the compressor output does not correspond to the width at the CPM output. The amplified pulse can be much shorter than the oscillator pulse.

At the output of our four-prism compressor we routinely obtain a single-shot measurement of a pulse width shorter than $60 \mathrm{fs}$. Figure 3 shows the autocorrelation trace of a 52-fs single pulse obtained without smoothing. This curve needs comment.

First, as one can see in Fig. 3, the autocorrelation function presents large wings. We believe that these wings do not arise from scattered UV but from the high-index prisms. We have recently computed the third-order group velocity dispersion introduced by these prisms, and we found that this dispersion is much larger for high-index prisms than for standard silica prisms and has an opposite sign from the gratings. The effect of this third-order dispersion is important for the extreme frequencies of the pulse spectrum. As a consequence the bad chirp compensation for these frequencies leads to wings in the compressed pulse. Furthermore, we have observed in our amplifier some gain saturation. This leads to a much higher gain for extreme frequencies of the spectrum amplifying the wings of the pulse; it could explain the experimental observations.

The second remark is about the asymmetry of the curve. We believe that this could be due to a group velocity dispersion effect in the autocorrelator. One of the two replicas that cross in the KDP crystal has

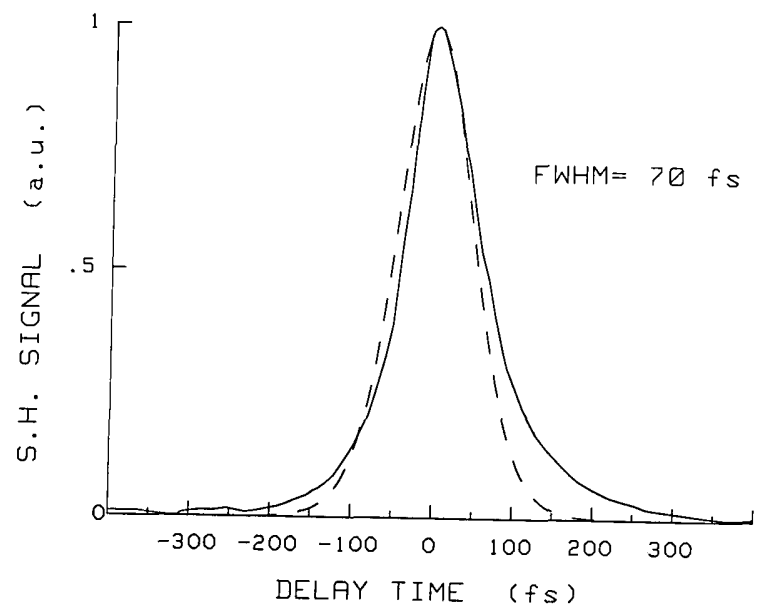

Fig. 4. Autocorrelation function averaged over fifty shots. The FWHM is $70 \mathrm{fs}$.

been reflected by the beam splitter while the other passes through the latter. As the spectrum of the pulse is not really symmetric, the temporal chirped pulse shape is asymmetric. Then the correlation function of an asymmetrically chirped pulse and a symmetric unchirped pulse can show some asymmetry. Figure 4 shows an averaging of fifty consecutive pulses giving an average FWHM of $\sim 70 \mathrm{fs}$. From shot to shot, we have observed variations of the FWHM from 50 to $80 \mathrm{fs}$. Generally, the shortest pulses are also the most powerful. At the present time, nobody has obtained single-pulse measurement of pulses directly produced by a CPM laser, so we do not know if these instabilities come from the CPM or the amplifier.

Using the optical Kerr effect in doped glass, ${ }^{15}$ we have recorded the third-order autocorrelation function of the amplified pulses. These recordings show nearly symmetric pulses with $\sim 80$-fs FWHM. We note that this value is in accordance with that obtained by averaging single-shot measurements.

It is difficult to estimate the actual time resolution of this kind of single-shot measurement. The first limitation comes from the same problems in classical autocorrelators: group velocity mismatch in the nonlinear crystal, dispersion effect in the autocorrelator,.... Other limitations come from the aberrations of the lens used to record the image of the crystal and from the size of a detector pixel. One can avoid this limitation by using a well-corrected UV lens system affording large magnification. We believe that, even with our simplified system, the time resolution is well under 50 fs.

Summarizing: in this paper we demonstrate that it is experimentally possible to measure a pulse width shorter than $60 \mathrm{fs}$ with a single pulse. Such a singleshot autocorrelation enables the optimization of dispersion compensation systems and the study of femtosecond amplified pulse stability. With our laser we found that the shortest pulses have a width of $\sim 50 \mathrm{fs}$ and an average width of $\sim 70 \mathrm{fs}$. The exact knowledge of a pulse's characteristics is useful for physicists who have to use such pulses. We predict that in the near 
future single-shot measurement systems will appear giving more information than does the spatial autocorrelator described here (peak power, chirp, asymmetry, ...).

We want to thank the Laboratoire d'Optique Appliquée (ENSTA, Palaiseau) for lending us an OMA I system and M. Dumont for helpful discussions. This work was partly supported by Direction des Recherches Etudes et Technique (Division Optique).

\section{References}

1. R. L. Fork, B. I. Greene, and C. V. Shank, "Generation of Optical Pulses Shorter than 0.1 ps by Colliding Pulse Mode-Locking," Appl. Phys. Lett. 41, 671 (1981).

2. R. L. Fork, C. V. Shank, R. Yen, and C. A. Hirlimann, "Femtosecond Optical Pulses," IEEE J. Quantum Electron. QE-19, 500 (1983).

3. A. Migus, A. Antonetti, J. Etchepare, D. Hulin, and A. Orszag, "Femtosecond Spectroscopy with High-Power Tunable Optical Pulses," J. Opt. Soc. Am. B 2, 584 (1985).

4. E. P. Ippen and C. V. Shank, "Techniques for Measurement," in Ultrashort Light Pulses (Springer-Verlag, Berlin, 1977), p. 83.

5. B. Colombeau, C. Froehly, and M. Vampouille, "Autocorrelation of Laser Pulses by Optical Processing of Fabry-Perot Spectrograms," Appl. Opt. 19, 534 (1980).
6. O. E. Martinez, "Linear Measurement of Ultrashort Light Pulses by Phase Contrast Optical Sweep," J. Opt. Soc. Am. B 2, 327 (1985).

7. P. Yeh, "Autocorrelation of Ultrashort Optical Pulses using Polarization Interferometry," Opt. Lett. 8, 330 (1983).

8. P. Sperber and A. Penzkofer, "Pulse Shape Determination of Intracavity Compressed Picosecond Pulses by Two Photons Fluorescence Analysis," Opt. Quantum Electron. 18, 145 (1986).

9. R. Wyatt and E. E. Marinero, "Versatile Single-Shot Background-free Pulse Duration Measurement Technique, for Pulses of Subnanosecond to Picosecond Duration," Appl. Phys. 25, 297 (1981).

10. J. Jansky, G. Conadi, and R. N. Gyuzalian, "On a Possibility of Analysing the Temporal Characteristics of Short Light Pulses," Opt.Commun. 23, 293 (1977).

11. R. N. Gyuzalian, S. B. Sogomonian, and Z. Gy. Horwarth, "Background-free Measurement of Time Behavior of an Individual Picosecond Laser Pulse," Opt. Commun. 29, 239 (1979).

12. Y. Ishida, T. Yajima, and A. Watanabe, "A Simple Monitoring System for Single Subpicosecond Laser Pulses using a S.H. Spatial Autocorrelation Method and a CCD Image Sensor," Opt. Commun. 56, 57 (1985).

13. F. Salin, P. Grangier, G. Roger, and A. Brun, "Observation of High-Order Solitons Directly Produced by a Femtosecond Ring Laser," Phys. Rev. Lett. 56, 1132 (1986).

14. F. Salin and A. Brun, "Dispersion Compensation for Femtosecond Pulses Using High-Index Prisms," J. Appl. Phys. 61, 4736 (1987).

15. J. Etchepare, G. Grillon, and A. Orszag, "Third Order Autocorrelation Study of Amplified Subpicosecond Laser Pulses," IEEE J. Quantum Electron. QE-19, 775 (1983).

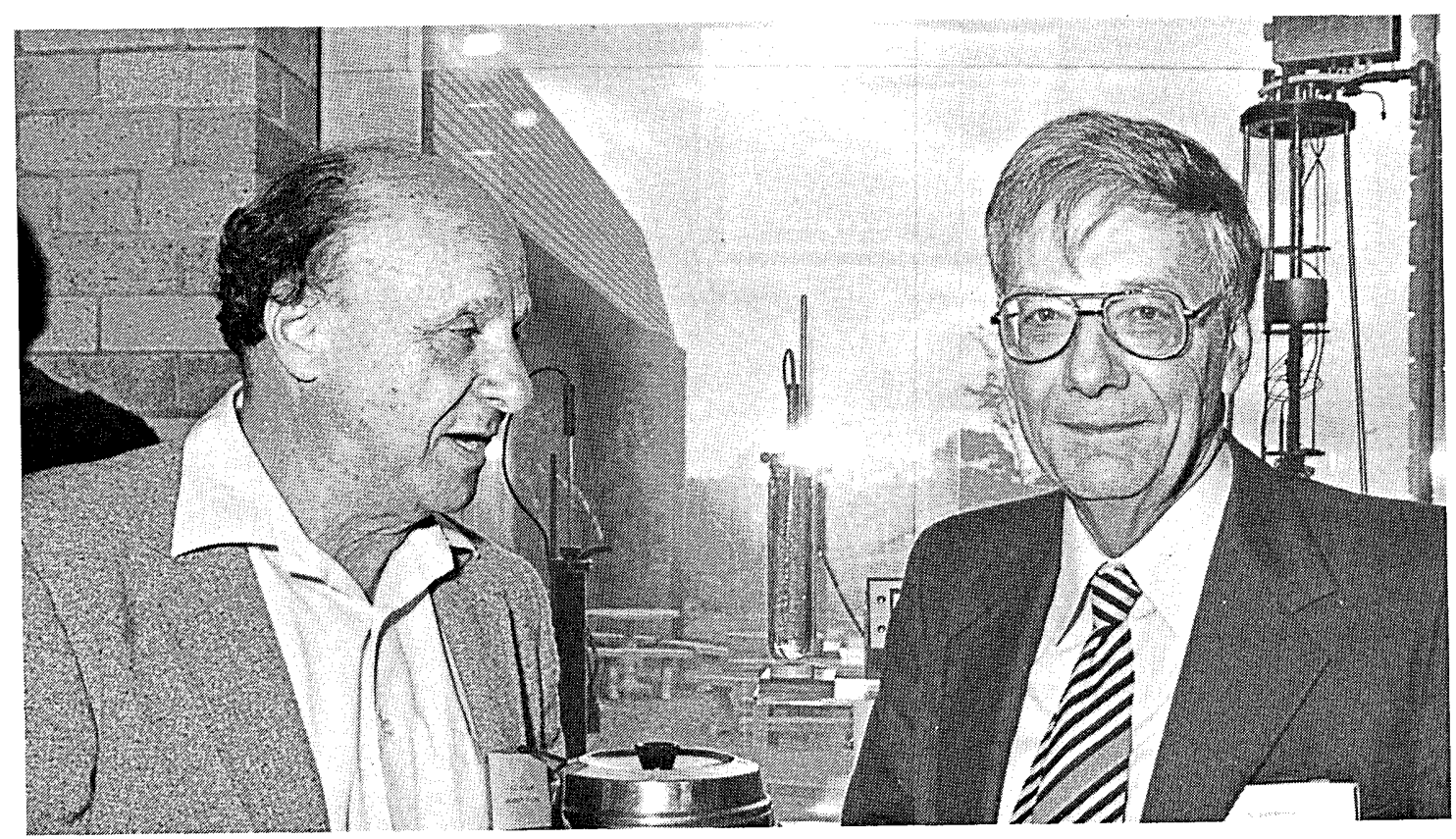

S. A. Friedland of Tel Aviv University (left) and N. Bloembergen. 